\title{
Large-scale velocity fluctuations in polar solar wind
}

\author{
B. Bavassano, R. Bruno, and R. D'Amicis \\ Istituto di Fisica dello Spazio Interplanetario (C.N.R.), Roma, Italy
}

Received: 21 May 2004 - Revised: 10 December 2004 - Accepted: 15 December 2004 - Published: 30 March 2005

\begin{abstract}
The 3-D structure of the solar wind varies dramatically along the Sun's activity cycle. In the present paper we focus on some properties of the polar solar wind. This is a fast, teneous, and steady flow (as compared to low-latitude conditions) that fills the high-latitude heliosphere at low solar activity. The polar wind has been extensively investigated by Ulysses, the first spacecraft to perform in-situ measurements in the high-latitude heliosphere. Though the polar wind is quite a uniform flow, fluctuations in its velocity do not appear negligible. A simple way to characterize the solar wind structure is that of performing a multi-scale statistical analysis of the wind velocity differences. The occurrence frequency distributions of velocity differences at time lags from 1 to $1024 \mathrm{~h}$ and the corresponding values of mean, standard deviation, skewness, and kurtosis have been obtained. A comparison with previous results in ecliptic wind at both low and high solar activity has been performed. It comes out that the kind of trend observed in the distributions for changing scale is the same for the different solar wind regimes. Differences between different flows just have an effect on the values of the distribution moments and the scales at which the transition from non-Gaussian to Gaussian-like behaviours occurs. This is typical of systems in which random fluctuations are mixed to coherent structures of some characteristic size, in other words, systems where long-range correlations cannot be neglected.
\end{abstract}

Keywords. Heliosphere (Solar wind plasma; Sources of the solar wind) - Space plasma physics (Turbulence)

\section{Introduction}

Plasma measurements by Ulysses during its first out-ofecliptic orbit (started after Jupiter's gravity assist in February 1992) have provided the first in-situ observations of the high-latitude solar wind. Two full orbits have now been completed (third aphelion on June 2004), covering an entire solar activity cycle. The data show that the 3-D structure of the solar wind varied dramatically over the solar cycle

Correspondence to: B. Bavassano

(bavassano@ifsi.rm.cnr.it) (e.g. McComas et al., 2003). Throughout the first orbit, in a period of low solar activity, the solar wind displayed quite a simple bimodal structure, with a persistently fast, tenuous and uniform solar wind at high heliographic latitudes (the so-called polar wind) and slower, more variable, and highly structured wind at low latitudes (e.g. McComas et al., 1998, 2000). In sharp contrast, around solar maximum variable flows were observed at all latitudes and the wind structure appeared to be a complicated mixture of flows coming from a variety of sources (McComas et al., 2002a, 2002b, 2003; Neugebauer et al., 2002). However, as highlighted by McComas et al. (2002b), this situation persists for a relatively short phase at high solar activity, whereas the remainder of the cycle appears dominated by a solar wind with bimodal structure.

A simple method to characterize the solar wind structure is that of looking at the statistical properties of the wind velocity variations at various scales. Under this perspective, multi-scale statistical analyses of velocity differences have been recently performed by Burlaga and Forman (2002) and Burlaga et al. (2003) for the ecliptic solar wind. The first study refers to observations at $1 \mathrm{AU}$ for both low and high solar activity, using Wind and ACE data during 1995 and 1999, respectively. The second one focuses on the radial evolution, by both applying a model for solar wind expansion and comparing to Voyager 2 data. Regarding the out-of-ecliptic solar wind, a comparison between wind velocity variations in high-latitude and in ecliptic solar wind for solar maximum conditions has been performed by Bavassano et al. (2004). The polar solar wind is the only wind regime not yet investigated through a multi-scale statistical analysis. Though polar wind is quite a uniform flow, fluctuations in its velocity magnitude $V$ are not negligible (e.g., see Neugebauer et al. (1995) on high-latitude microstreams). Thus, a multiscale analysis of the $V$ variations in polar wind appears as a useful tool to improve our understanding of this kind of plasma flow. This is the goal of the present study. We will analyse large-scale variations, defined (Burlaga, 1984) as those appearing in time profiles of hourly averages for intervals of several solar rotations (or, in terms of spectral analysis, periods roughly from a few hours to a solar rotation). 


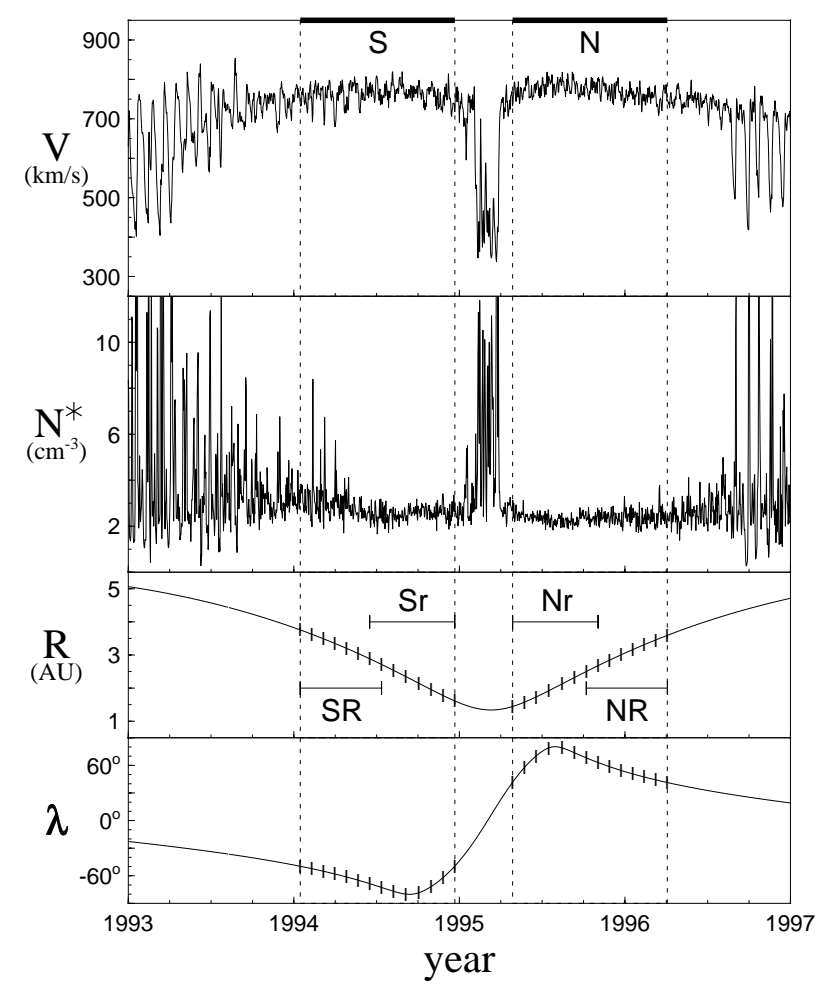

Fig. 1. From top to bottom, daily averages of the solar wind velocity $V$, of the proton number density $N^{*}$ (normalized to $1 \mathrm{AU}$ ), and of the Ulysses heliocentric distance $R$ and heliographic latitude $\lambda$ are plotted versus time for years 1993 to 1996 . Thick bars on top and dashed vertical lines highlight the analysed intervals during the south (S) and north (N) polar pass of the first out-of-ecliptic orbit. See text for further details.

\section{Data and method of analysis}

Solar wind measurements by the SWOOPS plasma analyzer aboard the Ulysses spacecraft (principal investigator D. J. McComas) have been used in the present analysis. From the individual velocity vectors, at a time resolution of 4 or $8 \mathrm{~min}$, depending on the mode of operation, hourly averages of the magnitude $V$ of the solar wind velocity have been computed. Our study is based on these hourly values.

An overview of the period under investigation is shown in Fig. 1 . Here daily averages of the wind velocity $V$ (in $\mathrm{km} / \mathrm{s}$ ), the proton number density $N^{*}$ (in $\mathrm{cm}^{-3}$, normalized to 1 astronomical unit assuming an inverse square scaling with distance), and the spacecraft coordinates (heliocentric distance $R$, in astronomical units, and heliographic latitude $\lambda$, in degrees) are plotted versus time for years 1993 to 1996, covering both southern and northern polar phases of the first outof-ecliptic orbit. Thick bars in the top panel and dashed vertical lines indicate the investigated intervals ( $\mathrm{S}$ and $\mathrm{N}$ ) from the southern and northern pass, respectively. Each interval corresponds to a high-latitude cut of the Ulysses orbit for a time length of thirteen solar rotations (as seen by the spacecraft). Solar rotations are shown in Fig. 1 by vertical ticks along the $R$ and $\lambda$ curves. Due to the asymmetry between the northern
Table 1. The analysed data intervals: start and end times (year, day, hour), minimum and maximum distances ( $R$, in $\mathrm{AU})$, and latitudes $\left(\lambda\right.$, in ${ }^{\circ}$, with $\mathrm{n}$ and $\mathrm{s}$ for north and south, respectively).

\begin{tabular}{|c|c|c|c|}
\hline \multirow{2}{*}{$\begin{array}{l}\text { inter- } \\
\text { val }\end{array}$} & \multirow[t]{2}{*}{ time } & $R$ & $n$ \\
\hline & & $\min \quad \max$ & $\min$ \\
\hline $\mathrm{S}$ & $9401505-9435605$ & $1.61-3.76$ & $49.7 s-80.2 s$ \\
\hline $\mathrm{N}$ & $9511812-9609422$ & $1.44-3.59$ & $41.3 n-80.2 n$ \\
\hline SR & $9401505-9419410$ & $2.72-3.76$ & $49.7 s-72.7 s$ \\
\hline $\mathrm{Sr}$ & $9416812-9435605$ & $1.61-2.89$ & $49.7 \mathrm{~s}-80.2 \mathrm{~s}$ \\
\hline $\mathrm{Nr}$ & $9511812-9530620$ & $1.44-2.67$ & $41.3 n-80.2 n$ \\
\hline NR & $9528101-9609422$ & $2.50-3.59$ & $41.3 n-68.2 n$ \\
\hline
\end{tabular}

and southern orbital leg, the same time length does not correspond to the same latitudinal range in the two hemispheres. In fact, in the Northern (Southern) Hemisphere 13 rotations correspond to latitudes poleward of $41.3^{\circ}\left(49.7^{\circ}\right)$. To look for the presence of radial trends, the $\mathrm{N}$ and $\mathrm{S}$ intervals have been further divided into 2 subintervals of 7 rotations each, referring to the inner and outer portion of the investigated intervals. They are indicated by segments drawn in the $R$ panel and labelled $\mathrm{Nr}$ and $\mathrm{Sr}$ (north and south inner regions) and NR and SR (north and south outer regions), respectively. Obviously, latitude changes as well with radial distance along the Ulysses trajectory, however, the latitudinal excursions in the inner and outer intervals are not strongly different, thus variations, if any, should be mainly due to radial effects. The beginning and end times of these intervals, together with their distance and latitude ranges, are given in Table 1 . It is worth noting that there is a partial overlapping between the inner and the outer intervals.

As shown by Fig. 1, in the selected intervals the solar wind velocity (top panel) is varying between $\sim 700$ and $\sim 800 \mathrm{~km} / \mathrm{s}$. These variations are much smaller than those typically observed in the ecliptic wind (for instance, see in the figure the large $V$ variations during the low-latitude pass of Ulysses at the beginning of 1995). However, polar wind variations do not appear negligible. Though weak, at large enough distances they can lead to appreciable effects. As already noticed by previous studies (e.g. Horbury and Balogh, 2001), at the time of Ulysses observations the southern wind velocity was more modulated by corotating features than the northern one, especially in the outer region. This is very well highlighted by the north-south difference seen in the $N^{*}$ panel for sharp density enhancements, typically built up by compression effects at velocity gradients.

The multi-scale statistical analysis of the velocity variations is based on 1) the computation of velocity differences at different time lags and 2) the evaluation of statistical quantities for the resulting ensembles (e.g. see Burlaga and Forman, 2002). Thus, starting from the time series of $V$ hourly averages, we have first derived a set of time series of velocity differences $d V n(t)$ at time lags $\tau=2^{n}$ (in hours) as

$d V n \equiv d V n(t)=V\left(t+2^{n}\right)-V(t)$ 
for $n=0,1,2, \ldots, 10$. Then, for each of the eleven $d V n$ ensembles, the occurrence frequency distribution and the corresponding values of mean, standard deviation, skewness, and kurtosis have been computed. All this provides an overview of the basic features of the solar wind velocity structure at scales from 1 to $1024 \mathrm{~h}$ (or, 0.0417 to 42.7 days). The choice of 10 as an upper value for $n$ is done to avoid a strong fall of the size of the $d V n$ ensemble. With a number of hourly averages for each of the analysed intervals $(\mathrm{Nr}, \mathrm{NR}, \mathrm{Sr}$, and $\mathrm{SR}$ ) in the range 4300-4500, at $n=10$ the number of data has dropped to $\sim 76 \%$ of that at $n=0$. If $n=11$ is used, this percentage abruptly decreases to $\sim 53 \%$.

As is well known, the skewness, the third moment normalized to the second moment raised to $3 / 2$, measures the asymmetry of a distribution, and the kurtosis, the fourth moment normalized to the squared second moment, measures its peakedness (relative to a Gaussian distribution). For a Gaussian distribution the skewness is obviously 0, while the kurtosis has a value of 3 . The definition used here gives an unbiased estimate of the kurtosis (essentially with subtraction of the factor 3), then in the present paper the kurtosis is 0 for a Gaussian distribution. In the following we will refer to these quantities as moments of a distribution, though they are not directly the moments but rather ratios between the moments. Note that some authors call flatness the ratio of the fourth moment to the squared second moment and define kurtosis as flatness -3 . A caveat about kurtosis in in order. Though widely used as a measure of non-Gaussianity, kurtosis has some drawbacks when estimated from measured samples (Hyvärinen and Oja, 2000). The main problem is that it can be very sensitive to outliers (Huber, 1985), in other words the value of kurtosis may depend on only a few observations in the tails of the distribution, which might be erroneous or irrelevant observations.

In addition to this kind of analysis, we have also computed power spectra of the $V$ fluctuations to obtain an overview of the scaling regime(s) and look for periodicities in the data. This has been made separately for the four intervals analysed (Nr, NR, Sr, and SR). In the following sections we will first examine the results of this spectral analysis, then we will focus on the distributions of the velocity differences and their moments.

\section{Power spectral density of the velocity fluctuations}

The power spectral density (PSD) of the velocity fluctuations has been computed by using an FFT algorithm on intervals of 4096 hourly averages (i.e. 170.67 days in time). These intervals, slightly shorter than the four selected intervals, have been chosen in such a way as to cover the innermost portion of $\mathrm{Nr}$ and $\mathrm{Sr}$ and the outermost portion of $\mathrm{NR}$ and SR, respectively. The results of the spectral analysis are shown in Fig. 2, where, for sake of simplicity, we use the labels $\mathrm{Nr}, \mathrm{NR}, \mathrm{Sr}$, and SR, though the time intervals to which the FFT analysis has been applied are not exactly coincident with those of Table 1 . For each panel the exact radial range and time interval

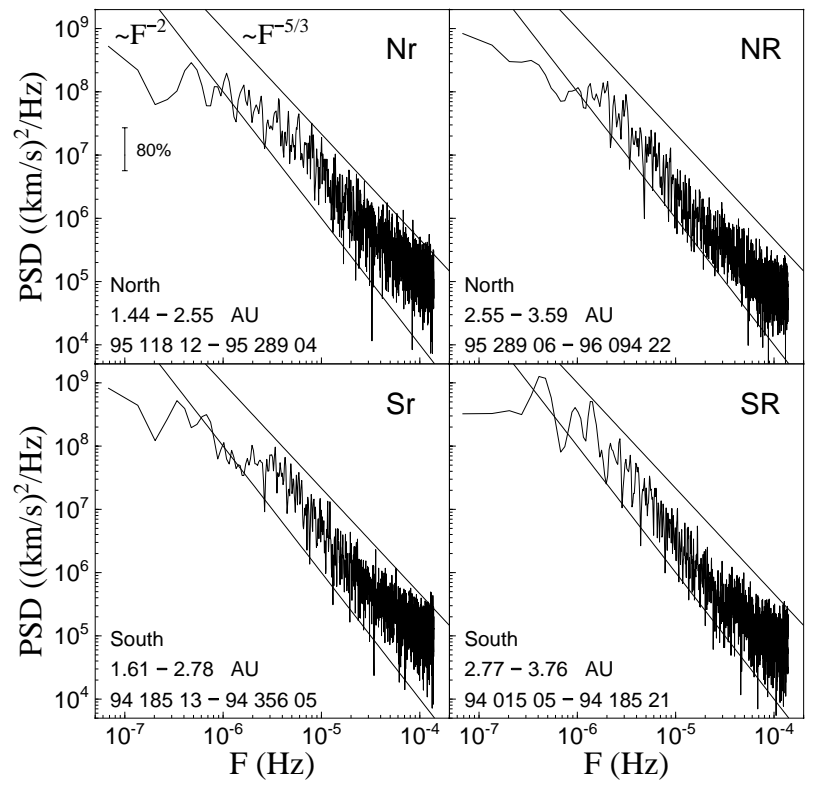

Fig. 2. Power spectral density (PSD) of the velocity fluctuations versus the frequency $F$. Panels $\mathrm{Nr}$ and $\mathrm{NR}$ ( $\mathrm{Sr}$ and SR) are for the inner and outer region of the northern (southern) polar wind, respectively. Radial range and time interval are indicated in each panel. Straight lines are drawn to indicate the $F^{-5 / 3}$ and $F^{-2}$ scalings. An $80 \%$ confidence bar is shown in panel $\mathrm{Nr}$.

are given at the bottom. A 3-pt smoothing procedure has been applied to the spectral estimates. An $80 \%$ confidence interval is shown in panel Nr.

Though similar in shape, the spectra of Fig. 2 exhibit differences in several respects. Apart from the lowest and the highest frequencies, the spectra appear well bounded by the $F^{-5 / 3}$ and $F^{-2}$ power laws. As is well known, the first corresponds to Kolmogorov's scaling for hydrodynamic turbulence in the inertial range, while the second is generally understood as a scaling due to a series of discontinuities. It is difficult to obtain a general conclusion about a favourite scaling with frequency. For instance, the $\mathrm{Nr}$ spectrum seems closer to $F^{-5 / 3}$, while $F^{-2}$ seems better for the SR spectrum. The high-frequency flattening observed for all the spectra is almost certainly related to instrumental sensitivity effects. Regarding low frequencies, the SR spectrum is characterized by the presence of peaks near and below $10^{-6} \mathrm{~Hz}$, that may be associated with solar rotation effects. In fact, for this interval the average duration of a solar rotation is 25.6 days, which corresponds to a frequency of $4.5 \times 10^{-7} \mathrm{~Hz}$. The largest peak in the SR spectrum is just around this frequency, and two other major peaks appear as harmonics of the solar rotation frequency. All this is not surprising. Figure 1 clearly shows that a residual modulation in the wind velocity is present for a major fraction of the SR interval, a highlatitude remnant of the corotating velocity structures seen during 1993 by Ulysses. 


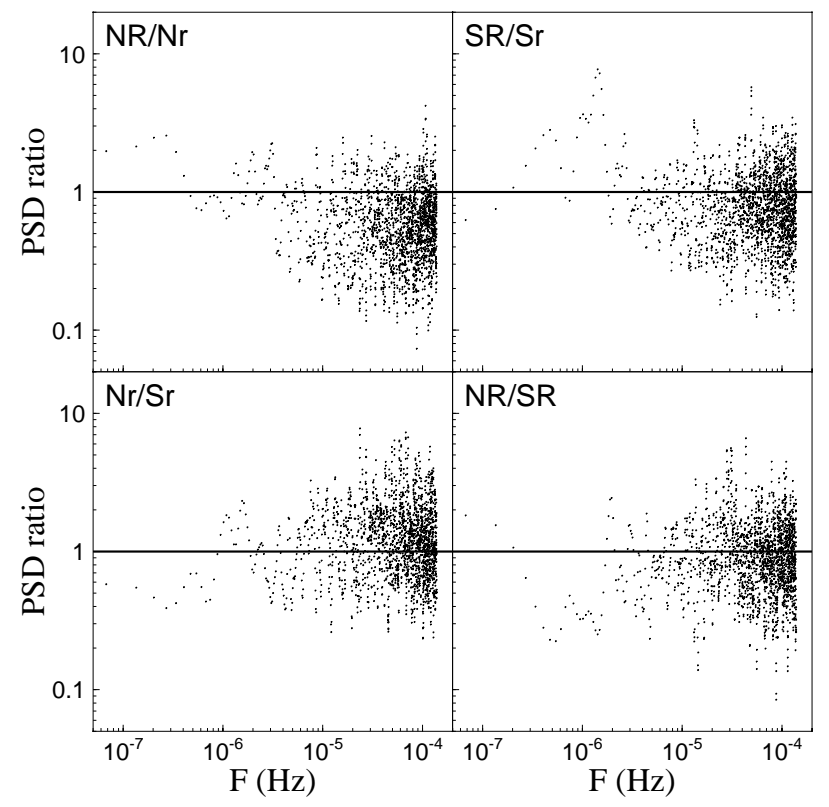

Fig. 3. Power density ratios are plotted to compare results in the analysed intervals. The upper panels refer to radial variations in the same hemisphere, while a comparison between the two hemispheres, for approximately the same radial range, is performed in the lower panels.

The level of power spectra in Fig. 2 appears to decrease when going from the inner ( $\mathrm{Nr}$ and $\mathrm{Sr}$ ) to the outer (NR and $\mathrm{SR})$ portion of the analysed intervals. As mentioned above, since the latitudinal excursion of the inner and outer intervals is almost the same, the observed power decline should be only due to a radial effect. To obtain an overall comparison between the power levels in the four different intervals we plot in Fig. 3 their mutual ratios. The upper panels refer to radial variations in the same hemisphere, while a comparison between the two hemispheres, for approximately the same radial range, is performed in the lower panels. The radial decrease just mentioned is clearly apparent in the ratios plotted in the upper panels, with values generally below unity (especially at North). Regarding the North-South differences, in the inner region the northern wind velocity seems slightly more variable than that of the corresponding southern wind. No clear trend comes out in the outer region. All this primarily holds for the core of the investigated frequency range. An example of a departure from these trends is the strong presence of solar rotation effects in the SR interval, as already discussed above.

\section{Statistics of the velocity differences}

The velocity differences $d V 1, d V 2, d V 4$, and $d V 6$ (at time lags of 2, 4, 16, and $64 \mathrm{~h}$, respectively) are plotted versus time in Fig. 4 (second to fifth panel from top) for a period of one solar rotation. The $V$ hourly averages from which the velocity differences have been derived are shown in the

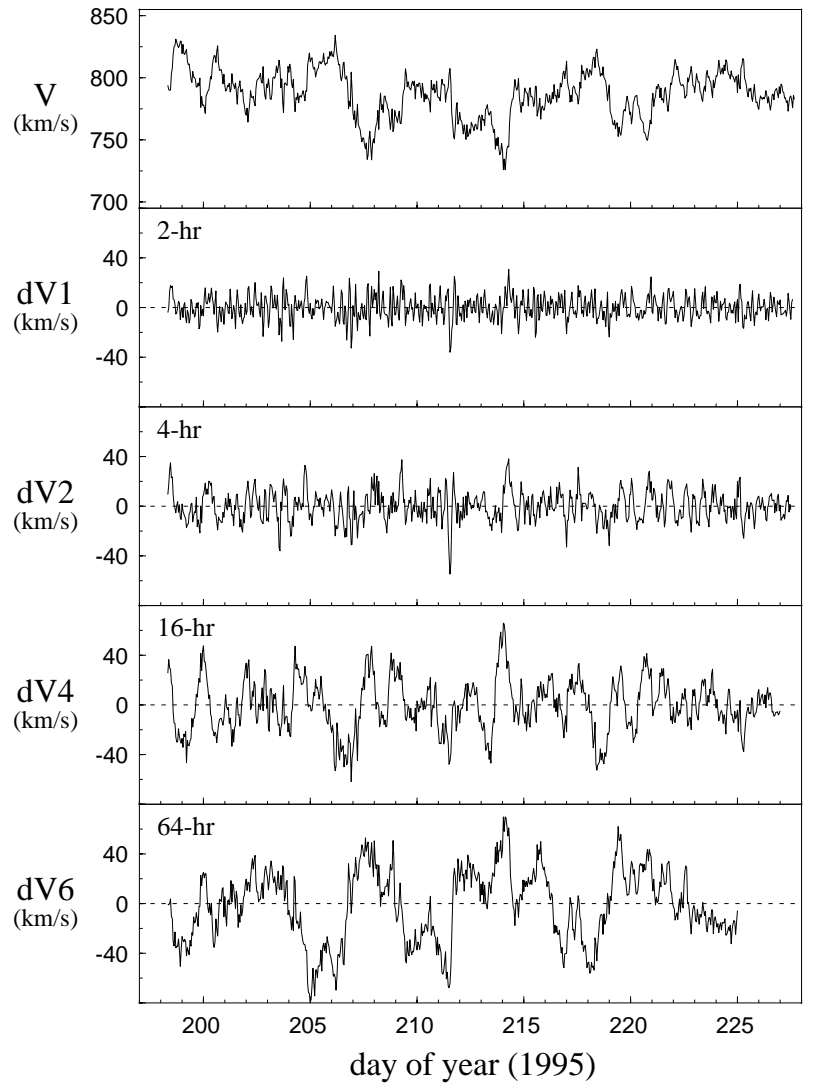

Fig. 4. Solar wind velocity $V$ and velocity differences $d V 1, d V 2$, $d V 4$, and $d V 6$ for a solar rotation at the top of the Ulysses' northern pass.

top panel. The selected interval is the fourth of the investigated solar rotations in the Northern Hemisphere (see Fig. 1), that encloses the highest latitudes $\left(\lambda\right.$ above $\sim 79^{\circ}$ ) of the Ulysses polar pass. The plotted set of curves clearly shows how at small scale the velocity differences have a relatively small amplitude and a turbulence-like appearance (e.g. see $d V 1$ panel), while at large scale (e.g. $d V 6$ panel) largeamplitude variations resembling the original $V$ pattern are observed.

Histograms of the velocity differences $d V 0, d V 1, d V 2$, $d V 3, d V 4$, and $d V 6$ (for time lags of 1, 2, 4, 8, 16, and $64 \mathrm{~h}$, respectively) are shown in the panels of Fig. 5. P is the occurrence frequency, namely the number of cases falling within each bin normalized to the total number. The bin width is $5 \mathrm{~km} / \mathrm{s}$ for all the scales. The curves drawn in each panel are Gaussian distribution functions computed by using the observed values of mean and standard deviation (in other words, they are not the result of a fit procedure). It is clearly seen that the distribution width increases when going towards longer lags. At a first glance the histograms appear exempt from strong non-Gaussian features.

A quantitative characterization of the $d V n$ frequency distributions can be obtained by evaluating their moments (or 


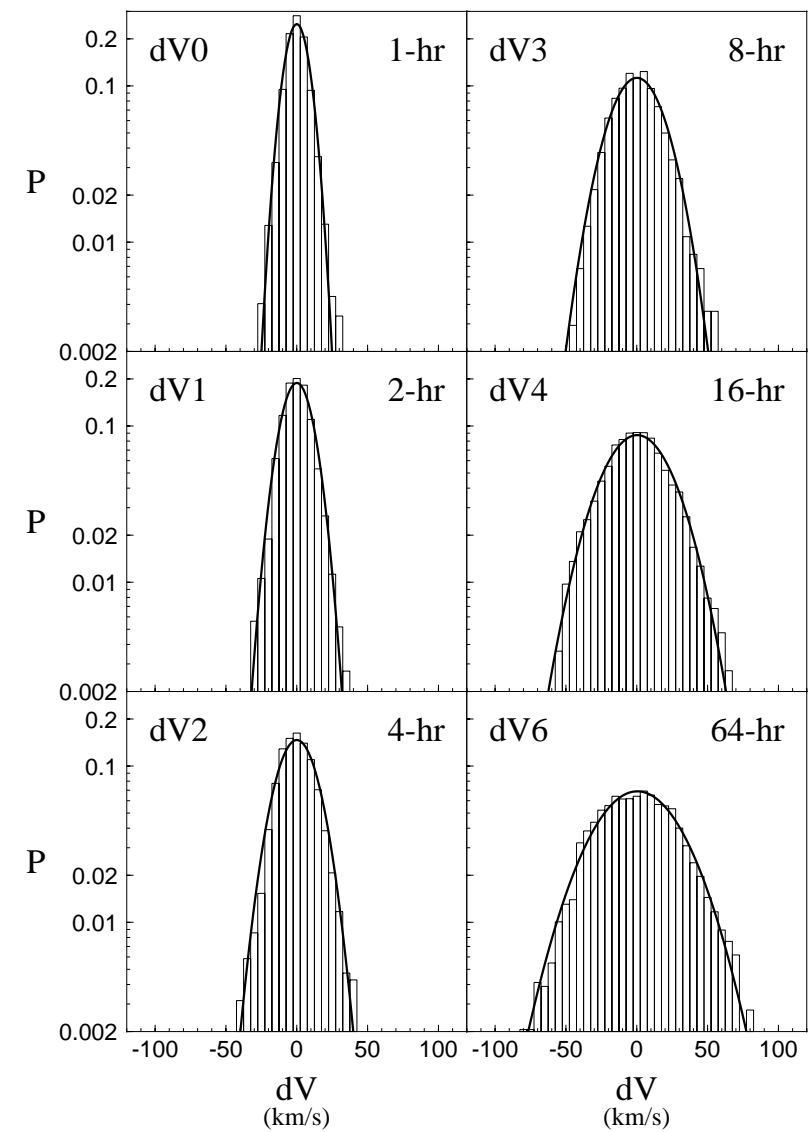

Fig. 5. The occurrence frequency $P$ (in a logarithmic scale) of the velocity differences $d V 0, d V 1, d V 2, d V 3, d V 4$, and $d V 6$ is shown by histograms in the different panels. The solid curves correspond to Gaussian distribution functions as computed from the observed values of mean and standard deviation.

moment-related parameters). In Fig. 6 the dependence upon $\tau$ of mean, standard deviation, skewness, and kurtosis is shown separately for the four (Nr, NR, Sr, and SR) investigated intervals. Regarding the mean (top panel), its value is seen to remain close to zero for all intervals up to $\tau=128 \mathrm{~h}$, then departures come out (that in some cases may be a nonnegligible fraction of the corresponding standard deviation). The standard deviation (second panel from top) roughly ranges from 5 to $45 \mathrm{~km} / \mathrm{s}$. For all the analysed intervals the standard deviation increases with $\tau$ for lags below $128 \mathrm{~h}$, then becomes nearly constant. These plateau levels are higher for the southern intervals, especially at large distance (see the SR curve). Finally, the values of skewness and kurtosis (third and fourth panel, respectively) clearly indicate that the statistics is different at different scale, with non-Gaussian distributions (i.e. departures from zero of skewness and/or kurtosis) at small lags and close-to-Gaussian distributions (i.e. nearly zero skewness and kurtosis) at large lags. This holds for all the examined intervals. The transition between the two kinds of distribution roughly occurs in the 16- to 32-h $\tau$ range. The largest departures from a Gaussian are observed

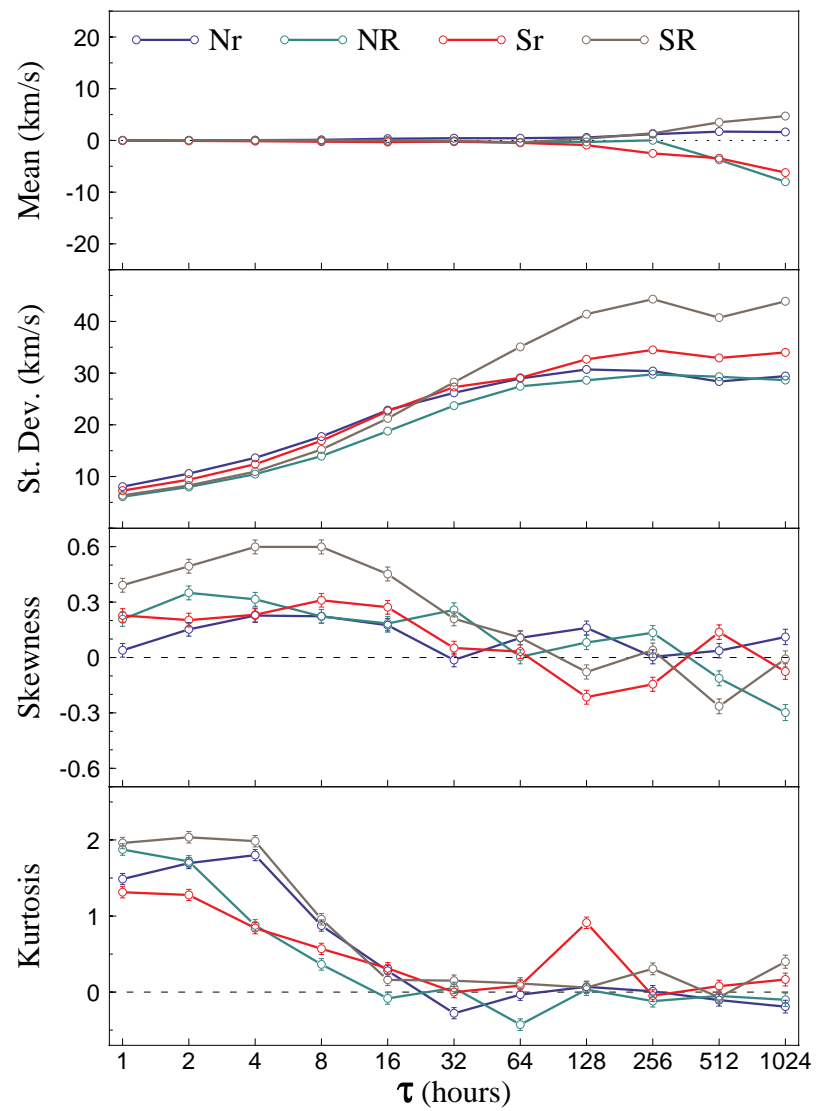

Fig. 6. The dependence of mean, standard deviation, skewness, and kurtosis on the time lag $\tau$ is shown separately for the four ( $\mathrm{Nr}, \mathrm{NR}$, $\mathrm{Sr}$, and SR) Ulysses intervals.

in the SR interval where, as already mentioned, the wind velocity exhibits a residual modulation by corotating structures.

Regarding the caveat given in Sect. 2 about kurtosis, a careful inspection of the data used in the analysis allows us to be confident that the observed kurtosis behaviour does not derive from erroneous or irrelevant data.

\section{Discussion and conclusion}

The 3-D structure of the solar wind is strongly dependent upon the phase of the solar activity cycle. The present study focuses on some properties of the polar solar wind. This is a fast, teneous, and relatively steady flow that, with exclusion of a rather short phase at high solar activity, is a dominant feature of the high-latitude heliosphere. Though small, fluctuations in the polar wind velocity cannot be considered negligible. In the present paper we have studied these fluctuations by using a multi-scale statistical analysis of the velocity differences at time scales from 1 to $1024 \mathrm{~h}$. This kind of technique has already been applied to ecliptic solar wind (Burlaga and Forman, 2002; Burlaga et al., 2003) and to high-latitude solar wind at solar maximum (Bavassano et al., 2004). In addition to this analysis, a classical tool such as 
power spectrum computation has been used in order to obtain an overview on scaling regimes and look for periodicities in the velocity data. In the following we will first discuss the spectral analysis results, then we will focus on the distributions of the velocity differences and their moments.

A comparison between our spectra (Fig. 2) and those obtained by Burlaga and Forman (2002) for ecliptic solar wind indicates that for frequencies above roughly $10^{-5} \mathrm{~Hz}$ the level of the velocity fluctuations is lower (of about one order of magnitude) in polar wind, though the spectral shape is similar. It must be noted, however, that polar observations refer to larger distances and that in the analysis of Burlaga and Forman (2002) the radial component of the solar wind velocity was used, instead of the velocity magnitude (as done here). At frequencies below $10^{-5} \mathrm{~Hz}$ the differences become more relevant. In fact, contrary to ecliptic wind spectra, polar wind spectra tend to flatten when approaching frequencies close to $10^{-6} \mathrm{~Hz}$ (with the exception of the SR interval, already discussed above). This spectral range, as highlighted by Burlaga and Forman (2002), reflects variations associated with the stream structure. It is not surprising that this frequency band appears depleted in polar wind. Finally, around and below $10^{-6} \mathrm{~Hz}$ the ecliptic spectra are dominated by peaks introduced by long-living (more than one solar rotation) structures rotating with the Sun. These peaks are absent in the polar wind, with the exception of the SR interval, where the solar modulation still has non-negligible effects.

Let us examine now the multi-scale analysis results, in particular the distribution moments and their variation with scale, or time lag $\tau$. We have found that 1 ) the mean values of the distributions remain close to zero up to $\tau=128 \mathrm{~h}$, then departures from zero come out, 2) the standard deviation increases with $\tau$ for lags below $128 \mathrm{~h}$, then becomes nearly constant, 3 ) the values of skewness and kurtosis show that non-Gaussian distributions are typical of the small lags, while close-to-Gaussian distributions are generally observed at large lags, and 4) the transition between these two kinds of distribution roughly occurs in the 16- to 32-h $\tau$ range.

When these results are compared to those by Burlaga and Forman (2002) for ecliptic wind at both high and low solar activity, it clearly appears that the overall trend is similar but with relevant differences in the values of the parameters (note that no comparison is possible for the distribution means, since they have not been reported by those authors). In all cases the standard deviation of $d V n$ distributions first increases with $\tau$ and then remains nearly constant. However, in the polar wind the standard deviation is notably lower than in the ecliptic wind. This simply reflects the fact that the polar wind is a much less variable flow than that observed at low latitudes. Regarding skewness and kurtosis, in all cases we have non-Gaussian distributions at small lags and Gaussianlike distributions at large lags. However, the departure from a Gaussian behaviour at small scales is much less pronounced for polar wind. For instance, for $\tau=2 \mathrm{~h}$, in the polar wind we find kurtosis values between roughly 1 and 2, while for ecliptic wind values as high as $\sim 16$ near solar maximum and $\sim 7$ near solar minimum are observed. Moreover, in the polar wind the Gaussian behaviour is established at smaller scales (roughly 1 day) than in the ecliptic wind (3 or more days). It is worth mentioning that our results agree very well, in terms of both scales and amplitudes, with those by Neugebauer et al. (1995) on the presence of microstreams in the polar wind.

In conclusion, the kind of trend observed in the $d V n$ distribution moments for changing scale is the same for different solar wind regimes as 1) the ecliptic wind near solar maximum, dominated by transient flows related to solar disturbances, 2) the ecliptic wind near solar minimum, dominated by fast streams from equatorward expansions of polar coronal holes, and 3) the polar wind, an almost steady flow from polar solar regions. The differences between flow regimes have rather the effect of leading to different values of 1) the distribution moments, and 2) the scales at which the transition from non-Gaussian to Gaussian-like behaviours occurs. All this is reminiscent of systems in which random fluctuations are mixed to coherent structures of some characteristic size (e.g. Bruno et al., 2003a, 2003b, 2004), in other words, systems in which long-range correlations cannot be neglected. In our case the wind velocity pattern represents the structure on which fluctuations, probably related to turbulent processes, are superposed.

Acknowledgements. The use of data from the Ulysses/SWOOPS plasma analyzer (principal investigator D. J. McComas, Southwest Research Institute, San Antonio, Texas, USA) is gratefully acknowledged. The data have been obtained through the NASA World Data Center A for Rockets and Satellites (Goddard Space Flight Center, Greenbelt, Maryland, USA). We are indebted to G. Consolini for very fruitful discussions. The present work has been supported by the Italian Space Agency (ASI) under contract IR/064.

Topical Editor R. Forsyth thanks R. Skoug for his help in evaluating this paper.

\section{References}

Bavassano, B., D'Amicis, R., and Bruno, R.: Solar wind velocity at solar maximum: A search for latitudinal effects, Ann. Geophys., 22, 3721-3727, 2004,

SRef-ID: 1432-0576/ag/2004-22-3721.

Bruno, R., Carbone, V., Sorriso-Valvo, L., and Bavassano, B.: Radial evolution of solar wind intermittency in the inner heliosphere, J. Geophys. Res., 108(A3), 1130, doi:10.1029/2002JA009615, 2003a.

Bruno, R., Carbone, V., Sorriso-Valvo, L., and Bavassano, B.: On the role of coherent and stochastic fluctuations in the evolving solar wind MHD turbulence: Intermittency, Proceedings of Solar Wind 10 Conference, edited by: Velli, M., Bruno, R., and Malara, F., American Institute of Physics Conference Proceedings 679, p. 453, doi:10.1063/1.1618632, 2003 b.

Bruno, R., Sorriso-Valvo, L., Carbone, V., and Bavassano, B.: A possible truncated-Lévy-flight statistics recovered from interplanetary solar-wind velocity and magnetic-field fluctuations, Europhys. Lett., 66, 146, doi:10.1209/epl/12003-10154-7, 2004.

Burlaga, L F.: MHD processes in the outer heliosphere, Space Sci. Rev., 39, 255-316, 1984. 
Burlaga, L. F. and Forman, M. A.: Large-scale speed fluctuations at 1 AU on scales from 1 hour to $\approx 1$ year: 1999 and 1995, J. Geophys. Res., 107(A11), 1403, doi:10.1029/2002JA009271, 2002.

Burlaga, L. F., Wang, C., Richardson, J. D., and Ness, N. F.: Evolution of the multiscale statistical properties of corotating streams from 1 to $95 \mathrm{AU}$, J. Geophys. Res., 108(A7), 1305, doi:10.1029/2003JA009841, 2003.

Horbury, T. S. and Balogh, A.: Evolution of magnetic field fluctuations in high-speed solar wind streams: Ulysses and Helios observations, J. Geophys. Res., 106, 15 929-15 940, 2001.

Huber, P.: Projection pursuit, Ann. Statistics, 13, 435-442, 1985.

Hyvärinen, A. and Oja, E.: Independent component analysis: algorithms and applications, Neural Networks, 13, 411-431, 2000.

McComas, D. J., Bame, S. J., Barraclough, B. L., Feldman, W. C., Funsten, H. O., Gosling, J. T., Riley, P., Skoug, R., Balogh, A., Forsyth, R., Goldstein, B. E., and Neugebauer, M.: Ulysses return to the slow solar wind, Geophys. Res. Lett., 25, 1-4, 1998.

McComas, D. J., Barraclough, B. L., Funsten, H. O., Gosling, J. T., Santiago-Muñoz, E., Skoug, R. M., Goldstein, B. E., Neugebauer, M., Riley, P., and Balogh, A., Solar wind observations over Ulysses first full polar orbit, J. Geophys. Res., 105, 10419 10433,2000
McComas, D. J., Elliot, H. A., and von Steiger, R.: Solar wind from high-latitude coronal holes at solar maximum, Geophys. Res. Lett., 29(9), doi:10.1029/2001GL013940, 2002a.

McComas, D. J., Elliot, H. A., Gosling, J. T., Reisenfeld, D. B., Skoug, R. M., Goldstein, B. E., Neugebauer, M., and Balogh, A.: Ulysses' second fast-latitude scan: Complexity near solar maximum and the reformation of polar coronal holes, Geophys. Res. Lett., 29(9), doi:10.1029/2001GL014164, 2002 b.

McComas, D. J., Elliott, H. A., Schwadron, N. A., Gosling, J. T., Skoug, R. M., and Goldstein, B. E.: The three-dimensional solar wind around solar maximum, Geophys. Res. Lett., 30(10), 1517, doi:10.1029/2003GL017136, 2003.

Neugebauer, M., Goldstein, B. E., McComas, D. J., Suess, S. T., and Balogh, A.: Ulysses observations of microstreams in the solar wind from coronal holes, J. Geophys. Res., 100, 23 389-23 395, 1995.

Neugebauer, M., Liewer, P. C., Smith, E. J., Skoug, R. M., and Zurbuchen, T. H.: Sources of the solar wind at solar activity maximum, J. Geophys. Res., 107(A12), 1488, doi:10.1029/2001JA000306, 2002. 